\title{
Podcast Generator and Pluriversiradio: An Educational Interactive Experience
}

\author{
Alberto Betella and Marco Lazzari \\ University of Bergamo, Faculty of Educational sciences, Piazzale Sant'Agostino, 2 \\ I-24124 Bergamo Italy \\ marco.lazzari@unibg.it
}

\begin{abstract}
This paper presents an open source podcast publishing application which has been implemented to create an educational podcasting service at the University of Bergamo (Italy) and has subsequently been adopted by several universities and other podcasters in Italy and abroad.
\end{abstract}

Keywords: educational podcasting, free software, open source, podcast publishing, distance education, distance learning, mobile learning.

\section{Introduction}

The use of podcasting is spreading throughout the education sector due to the increasing popularity of mobile media players. Podcasting is becoming an interesting and attractive way for distributing educational materials [1, 2], and several schools and universities have set up podcasting services [3,4]. This paper presents an open source software, Podcast Generator (PG), which comprises a set of web based tools, that allow users to upload audio or video media files via a web form along with information (e.g. description, keywords) and create a podcast feed. PG was developed to implement Pluriversiradio, a podcasting service at the University of Bergamo. ${ }^{1}$

The first version of PG was designed in September 2005, when podcasting was still an unknown technology to the majority of the internet users. In the design process we considered to start improving some existing podcast-publishing applications. Our research did not produce successful results; besides a plug-in which needed a full installation of the Wordpress ${ }^{2}$ blog software to work, we found two scripts which could be adopted to comply our aim The first one, PodAdmin, ${ }^{3}$ consisted of a simple interface which allowed to upload mp3 files and created a basic XML feed, but lacked important functionalities such as file renaming on upload in case of duplicated files. The second one was a php script ${ }^{4}$ which allowed to create a RSS feed reading a folder on a server where the user had previously uploaded mp3 files, but some major features such as password protection were not available. Moreover, we contacted Apple to receive more information about iTunes $U$, the educational podcasting platform adopted by some universities in the USA, but they replied that the service was not available at that time outside the USA and Canada.

\footnotetext{
${ }^{1}$ Web site: http://www.pluriversiradio.it - Feed: http://www.pluriversiradio.it/feed.xml

${ }^{2}$ http://wordpress.org/

${ }^{3} \mathrm{http}: / /$ podadmin.sourceforge.net/

${ }^{4}$ http://www.ontology.com/canton/projects/podcasting-script.php
} 
Therefore, we started designing our own application and released it under free GNU/GPL license in order to share our work with the open source community. Our main goal was creating an easy to use, yet powerful, web based software to allow any user, even without programming experience and knowledge, to create a podcasting service in a few steps and automatically generate a w3c-compliant XML feed. To achieve this goal the script had to feature an easy setup process and an interactive interface to let the user communicate with the server in a transparent way. The practical implementation of PG started on February 2006: the software was written in PHP and was officially released on Sourceforge.net, the world's largest open source software development web site, on April 2006. Pluriversiradio, which is based on PG, has been used during the second semester of the year 2005-2006 for courses on multimedia communication at the University of Bergamo; after those first experiments, other lecturers began recording podcasts for their students. Quantitative and qualitative analyses of exam results and satisfaction surveys highlight that involving students in producing podcasts had positive effects on learning [5].

\section{Features}

Podcast Generator features an automatic setup wizard which allows to install the script on an http server in a very short time and without any technical knowledge of PHP; after a guided setup the user is able to handle the administration (uploading, editing, deleting episodes) without further configuration. PG supports 21 media file types (mp3, ogg, m4v, mov, etc.) and web mp3 streaming, and generates XML feeds, meeting the w3c standards and supporting iTunes-specific tags; it provides a password protected administration interface to manage episodes and customize the script and builds dynamic 'All Podcasts' and 'Recent Podcasts' web pages.

PG is especially suitable for podcasters who do not have technical knowledge but wish to use podcasting in their classes. It is also suitable for experienced podcasters who want to save time and avoid the risk of syntax errors when dealing with the RSS feed. The setup wizard and the whole program interface are available in twelve languages ${ }^{5}$ and several volunteers are working on other translations.

\section{Architecture}

The software architecture works on different key levels we assume as "modules". These modules operate on the server-side and are processed by the PHP engine which generates the web page for the client.

\subsection{Module 1: Database}

Podcast Generator and Pluriversiradio are based either on a flat CSV file and on a MySQL database to store episodes data and information. We oriented our choice to maintain the $\mathrm{CSV}$ feature in order to keep low server requirements to run the

${ }^{5}$ Catalan, Chinese, Danish, Dutch, English, French, German, Italian, Japanese, Portuguese, Romanian, Russian. 
program: in order to install PG, just a server with PHP support is needed and no MySQL is required. On uploading or editing every episode, a description file containing data provided by the user (such as title, description, keywords, etc.) is generated and associated to the uploaded media file. On the other hand, Pluriversiradio features a MySQL database to store episodes data and operate complex search aimed at reducing the number of downloads and saving bandwidth.

\subsection{Module 2: Web-Based Interface and User Interaction}

The web based administration interface of Podcast Generator does not require the user to have any technical knowledge. It is based on HTML and Javascript, so that the browser can validate some input fields before they are sent to the server. The interface also features a colored output which displays in green every successful action and in red any possible errors, so that users are given an immediate visual message. In case of server-related problems, PG will output some server configuration parameters (e.g. 'max_upload_size') which can help to understand the problem.

The use of a combination of HTML and Javascript allows to simplify the interface and display just the essential dialog boxes, showing the optional fields on demand: the user is required to fill just two fields (title and short description) in the file upload form; anyway it is possible to check 'add extra information' and a set of new options will appear on the web page: a long description, an image, keywords and author's data can be provided. This implies a targeted user experience towards what really is needed, avoiding displaying several forms to be filled in order to upload a single episode. Fields in the administration interface offer hints to help users understand the function of every choice and show guidelines to the data required. The interaction is easy and users are given access to the PHP engine through a simple back-end layer.

\subsection{Module 3: PHP Engine}

Podcast Generator's PHP engine processes users' input and creates dynamic web pages showing the recent podcasts, an archive containing a list of all episodes and a detailed 'permalink' page for each audio or video file. It also generates the XML podcast feed and re-creates it automatically on new uploads or modifications through the administration interface. The PHP engine performs multiple internal controls that check input text and correct it automatically. For instance, if the user uploads files containing non-valid characters, they will not be rejected but automatically corrected (e.g. spaces are replaced by underscores).

\subsection{Module 4: Security}

The administration interface is secured by a password; the user and password feature is handled by sessions, a PHP native feature, that assigns a unique session ID to the user and allows to preserve certain data across subsequent accesses. This licenses the user to maintain the identity and avoids multiple password requests during the same session. The software also performs multiple controls on variables passed through the GET method in the pages URLs. Every variable is double-checked and in case of file inclusion into the web page, an effective existence control is performed in order to avoid cross site scripting attacks. 


\section{Current Results}

Podcast Generator has been downloaded more than 14,000 times from Sourceforge servers in its first year and is currently used by at least 500 podcasting services, such as the Department of Neurological Sciences of the University of Bari (Italy), the universities of Aarhus (Denmark), Delaware (USA), Central Queensland (Australia) and Caen Basse-Normandie (France). ${ }^{6}$

The quick diffusion of PG is due to its efficiency, ease of use and installation: the open source nature of the program allowed the cooperation of a set of kind users, who translated the whole software. Moreover, the Dutch foundation Kennisnet Ict op School used PG for publishing a screencast series about podcasting with open source and promoted the use of our software in schools: for this purpose they have also developed a video podcast that shows the installation procedure of $\mathrm{PG}^{7}$

\section{References}

1. Brittain, S., Glowacki, P., Van Ittersum, J., Johnson, L.: Podcasting Lectures. EDUCAUSE Quarterly 3, 24-31 (2006)

2. Cambell, G.: There's Something in the Air: Podcasting in Education. EDUCAUSE Review 40(6), 32-47 (2005)

3. Frydenberg, M.: Principles and Pedagogy: the Two P's of Podcasting in the Information Technology Classroom. In: Proc. of the 23rd Annual Conf. for Information Systems Educators ISECON 2006, Dallas, TX (2006)

4. Malan, D.: Podcasting Computer Science E-1. In: Proc. of the 38th ACM Technical Symp. on Computer Science Education SIGCSE '07, Covington, KY (2007)

5. Lazzari, M.: Podcasting in the Classroom: Involving Students in Creating Podcasted Lessons. In: Proc. of the Conf. HCI Educators HCIEd 2007, Aveiro, Portugal (2007)

\footnotetext{
${ }^{6}$ http://www.neuroscienze.uniba.it/; http://www.media.au.dk/podcast/; http://admissions.udel.edu/uwired/; http://streaming.cqu.edu.au/podcast/; http://www.ingenium.unicaen.fr/podcastgen/

${ }^{7}$ http://files.ictopschool.net/podos/mp4/09.mp4
} 